\title{
Demandas Tecnológicas, Académicas y Psicológicas en Estudiantes Universitarios durante la Pandemia por COVID-19
}

\author{
Technologic, Academic, and Psychological Demands in University Students \\ during COVID-19 Pandemic
}

\author{
Adam Rosario-Rodríguez ${ }^{1}$, Juan Aníbal González-Rivera ${ }^{2}$, Andrés Cruz-Santos ${ }^{3}$ \& Luismiguel Rodríguez- \\ Ríos 4 \\ 1 Carlos Albizu University, San Juan, Puerto Rico. (D) https://orcid.org/0000-0003-1603-5040 \\ 2 Ponce Health Sciences University, Ponce, Puerto Rico. (D) https://orcid.org/0000-0003-0622-8308 \\ 3 Carlos Albizu University, San Juan, Puerto Rico. (D) https://orcid.org/0000-0002-6071-8693 \\ 4 Carlos Albizu University, San Juan, Puerto Rico. (iD https://orcid.org/0000-0001-7612-4745 \\ * Correspondencia: dr.adamrosario@gmail.com
}

Recibido: 8 junio 2020 | Aceptado: 29 junio 2020 | Publicado: 15 julio 2020

WWW.REVISTACARIBENADEPSICOLOGIA.COM

\section{Citar como:}

Rosario-Rodríguez, A., González-Rivera, J. A., Cruz-Santos, A., \& Rodríguez-Ríos, L. (2020). Demandas tecnológicas, académicas y psicológicas en estudiantes universitarios durante la pandemia por COVID-19. Revista Caribeña de Psicología, 4(2), 176-185. https://doi.org/10.37226/rcp.v4i2.4915

\section{RESUMEN}

La presente investigación examinó las demandas tecnológicas, académicas, y psicológicas experimentadas por una muestra de 167 estudiantes universitarios activos de 17 instituciones de educación superior en Puerto Rico durante el periodo de mayo-junio de 2020 durante la pandemia por COVID-19. La muestra estuvo compuesta mayormente por mujeres (81.4\%) con edades que fluctuaron entre $\operatorname{los} 18$ a los 61 años $(M=30.36, D E=11.46)$. Se utilizó un diseño no experimental transversal exploratorio-descriptivo. Los hallazgos principales indican que los estudiantes tuvieron problemáticas relacionadas a estresores por la sobrecarga de tareas, mala implementación de cursos en línea, falta de orientación de sus universidades, poca flexibilidad de sus profesores, entre otras. Estos hallazgos abren paso al estudio de los cambios de modalidades educativas (presencial a en línea) repentinas y su impacto en los estudiantes.

Palabras Claves: COVID-19; educación superior; cursos en línea; cambio de modalidad; estudiantes; universidad

\begin{abstract}
This research examined the technologic, academic, and psychological demands, experimented by a sample of 167 active university students from 17 institutions of higher education in Puerto Rico during may-june of 2020 during the pandemic of COVID-19. The sample was composed mainly by women (81.4\%) with ages between 18 to $61(M=$ $30.36, D E=11.46)$. A cross-sectional non-experimental exploratory-descriptive design was implemented. The principal results show the problems related to stressors for assignments overload, bad implementation of online courses, lack of orientation from their universities, poor flexibility from their professors, among others. These
\end{abstract}


results open the way to study the sudden changes of educational modalities (face-to-face to online) and their impact in students.

Keywords: COVID-19; higher education; online courses; changes in educational modalities; students; university

\section{INTRODUCCIÓN}

El SaARS-Cov-2 es un nuevo virus perteneciente a la familia de los coronavirus que fue detectado en diciembre de 2019 en Wuhan, una ciudad de la provincia de Hubei en la República Popular China (Yin \& Wunderink, 2018). Este nuevo virus induce la enfermedad infecciosa denominada COVID-19, la cual se propaga, principalmente, a través de secreciones respiratorias y contacto directo ( $\mathrm{Li}$ et al., 2020). Si bien los síntomas asociados al COVID-19 en su mayoría son leves (e.g., fiebre, tos, fatiga, dolor de garganta, entre otros), existen factores de riesgo (e.g., edad, sistema inmunológico comprometido, entre otros) que pueden desencadenar una infección aguda del tracto respiratorio (Wang, Xu et al., 2020; Wang, Zhang et al., 2020).

A la fecha, se estima en más de 11.5 millones los casos de personas infectada con SARS-CoV-2 (COVID-19) en todo el mundo; mientras que la cifra global de muertes sobrepasa los 538,000 y la de los recuperados supera los 6.3 millones de personas (BBC, 2020). A tales efectos y dado a la rápida propagación del COVID-19 a nivel global, así como a la inadecuada capacidad de respuesta y la falta de compromiso individual (e.g., uso de masacrilla) se han adoptado medidas sanitarias y cautelares sin precedentes, que afectan a diversos sectores, entre ellos la educación superior.

\section{La Educación Superior en Tiempos de COVID-19}

Conforme a la propagación del COVID-19 y las medidas cautelares implementadas a nivel global, el uso de modelos de enseñanza no convencionales en los sistemas de educación superior se ha convertido en un recurso indispensable para el cumplimiento de objetivos y responsabilidades instruccionales (Regmi \& Jones, 2020). En este contexto, el uso de tecnologías de la información y comunicación (TIC) para promover la enseñanza y el aprendizaje a distancia (e-learning) ha mostrado ser una poderosa herramienta. Esta permite incrementar la calidad de los procesos pedagógicos a distancia y la adaptación del contenido instruccional a las necesidades educativas de la comunidad académica en periodos de gran demanda como, por ejemplo, crisis socioeconómicas, desastres naturales o pandemias (Area \& Adell, 2009; Saini, 2020). Dentro de la flexibilidad y oportunidades que ofrece el aprendizaje mediado por TIC, la literatura destaca la posibilidad de construir ambientes de enseñanza que permiten: (1) incrementar la autonomía y responsabilidad del estudiante en su propio proceso de aprendizaje; (2) superar las limitaciones provocadas por la separación en espacio y tiempo; (3) acceder a multiplicidad de fuentes en cualquier momento; y (4) aprendizaje colaborativo (Landeta, 2007; Mezzadra \& Bilbao, 2010). Estas oportunidades proveen el escenario idóneo para el desarrollo de un proceso instruccional dinámico y participativo (Páez-Barón et al., 2015) centrado en las necesidades y diferencias individuales de la comunidad académica, principalmente en la de los estudiantes (Murphy et al., 2011).

Sin embargo, bajo la coyuntura del COVID-19, las demandas académicas, tecnológicas y psicológicas (producto de la implementación abrupta de modelos de aprendizaje a distancias y dificultades en el desarrollo de políticas de implementación y capacitación eficientes) han aportado a que el estudiante universitario experimente dificultades y barreras, independientemente de tener una actitud positiva hacia el uso de TIC en el proceso de enseñanza y aprendizaje (Sanz et al., 2020). A tales efectos, organizaciones internacionales como el Banco Interamericano de Desarrollo (2020) y la UNESCO (2019) exhortan a realizar un acercamiento desde lo conceptual y teórico con el objetivo de justificar las acciones, procedimientos y rutas que se deben tomar en beneficio de la comunidad académica, en especial hacia los estudiantes (Monasterio \& Briceño, 2020). Esto, con el propósito de identificar factores que incidan en la buena práctica del aprendizaje a distancia y permitan optimizar los resultados futuros; en momentos donde las soluciones adoptadas dependen de las capacidades, recursos y modalidades instruccionales con las que cuenta cada país y sus sistemas de educación superior. 


\section{Dificultades Presentadas por Estudiantes Durante} la Pandemia

El cambio abrupto en la modalidad de enseñanza y aprendizaje generado por la propagación de COVID-19 representa un impacto inmediato en las dificultades subyacentes que experimentan los estudiantes en el sistema de educación superior (Regmi \& Jones, 2020). A tales efectos, estudios recientes sugieren que el aislamiento social, la ansiedad y depresión relacionada al COVID-19, la dificultad para adaptarse a las nuevas tecnologías, las expectativas de mantener un horario y escenario académico regular, aspectos económicos (e.g., pago de matrícula y asistencia económica), el adiestramiento eficiente y la accesibilidad a recursos tecnológicos son las principales dificultades o preocupaciones experimentadas por los estudiantes durante la pandemia (Instituto Internacional para la Educación Superior en América Latina y el Caribe, 2020; Regmi \& Jones, 2020; UNESCO, 2020; Uprichard, 2020).

Dentro de los factores que promueven este escenario podemos destacar barreras relacionadas con la tecnología, falta de soporte técnico, analfabetismo digital, sistemas informáticos pobres y obsoletos y problemas de conectividad, así como el poco espacio de adaptación que el estudiantado ha tenido para reorganizarse y ajustarse a la pérdida de contacto social y las rutinas de socialización que formaban parte de su cotidianidad (Balluerka-Lasa, 2020; RISE, 2020). Este escenario, da lugar a grandes preocupaciones dada la posibilidad de que se genere un periodo de vulnerabilidad que promueva el abandono académico a gran escala (UNESCO, 2020). Dicho planteamiento promueve el desarrollo de proyectos investigativos como el presente, dirigidos a identificar rutas y elementos que faciliten la buena práctica e implantación eficiente de modelos de aprendizaje en momentos de gran demanda.

\section{Propósito del Estudio}

Tomando como punto de partida la revisión de literatura realizada, y el número limitado de investigaciones, el objetivo principal de esta investigación fue examinar las demandas tecnológicas, académicas y psicológicas que puedan estar experimentando los estudiantes universitarios en Puerto Rico durante la pandemia por COVID-19.

\section{MÉTODO}

\section{Diseño de Investigación}

Se utilizó un diseño no experimental transversal exploratorio-descriptivo (Hernández-Sampieri \& Mendoza, 2018). Esto con la finalidad de poder tener un panorama general de las demandas psicológicas y tecnológicas que han experimentado una muestra de estudiantes de diversas universidades en Puerto Rico durante la pandemia por COVID-19. La investigación fue aprobada por el IRB de Ponce Health Sciences University (protocolo 2005037702). La recopilación de datos se hizo durante el periodo de mayo-junio 2020 a través de la plataforma de PsychData. La investigación fue promocionada por Facebook. Incluía un mensaje explicando el propósito del estudio y los criterios de inclusión, así como un enlace de acceso al estudio de PsychData. Todos los participantes tuvieron acceso a un consentimiento informado el cual debían aceptar antes de tener acceso a los instrumentos de investigación.

\section{Participantes}

La muestra de esta investigación fue de 167 estudiantes universitarios matriculados en 17 universidades diferentes en Puerto Rico con edades que fluctuaron entre los 18 a los 61 años $(M=30.36, D E=11.46)$. Estuvo compuesta mayormente por mujeres $(81.4 \%$, $n=136)$, heterosexuales $(80.8 \%, n=135)$, solteros $(47.9 \%, n=80)$, cursando un grado de Maestría al momento del estudio $(53.3 \%, n=89)$, con una preparación académica de Bachillerato $(52.7 \%, n=88)$, desempleados $(44.9 \%, n=75)$, con un ingreso aproximado anual entre $\$ 0.00$ a $\$ 10,000(31.7 \%, n=53)$, y no son practicantes activos de alguna religión $(59.3 \%, n=99)$. Las personas que indicaron que practican activamente alguna religión mencionaron pertenecer en su mayoría al catolicismo $(16.8 \%, n=28)$. La mayoría considera que su religión influye positivamente en su estilo de vida $(35.9 \%, n=60)$. Los estudiantes que indicaron que sí trabajaban lo hacen mayormente en el sector de la educación $(23.4 \%, n=39)$. Se les preguntó a los participantes si tenían hijos a lo que la mayoría respondió que no $(74.3 \%, n=124)$. De los participantes que sí tienen hijos, la mayoría indicó que viven con ellos $(79.1 \%, n=34)$ y que estudian en universidades privadas $(23.3 \%, n=10)$. La Tabla 1 presenta todos los datos sociodemográficos de la muestra. 
Tabla 1

Datos Sociodemográficos.

\begin{tabular}{|c|c|c|}
\hline & $f$ & $\%$ \\
\hline \multicolumn{3}{|l|}{ Sexo } \\
\hline Mujer & 136 & 81.4 \\
\hline Hombre & 30 & 18.0 \\
\hline Otro & 1 & 0.6 \\
\hline \multicolumn{3}{|l|}{ Orientación Sexual } \\
\hline Heterosexual & 135 & 80.8 \\
\hline Gay & 9 & 5.4 \\
\hline Lesbiana & 4 & 2.4 \\
\hline Bisexual & 12 & 7.4 \\
\hline Pansexual & 2 & 1.2 \\
\hline Asexual & 2 & 1.2 \\
\hline Otro & 3 & 1.8 \\
\hline \multicolumn{3}{|l|}{ Estado Civil } \\
\hline Soltero/a & 80 & 47.9 \\
\hline Con pareja conviviendo & 21 & 12.6 \\
\hline Con pareja no conviviendo & 29 & 17.4 \\
\hline Casado/a & 32 & 19.2 \\
\hline Divorciado/a & 3 & 1.8 \\
\hline Separado/a & 2 & 1.2 \\
\hline \multicolumn{3}{|l|}{ Nivel Universitario que Cursa } \\
\hline Grado Asociado o Técnico & 4 & 2.4 \\
\hline Bachillerato & 41 & 24.6 \\
\hline Maestría & 89 & 53.3 \\
\hline Doctorado o equivalente & 32 & 19.2 \\
\hline Post Doctorado & 1 & 0.6 \\
\hline \multicolumn{3}{|l|}{ Ingreso Anual } \\
\hline$\$ 0$ a $\$ 10,000$ & 53 & 31.7 \\
\hline$\$ 11,000$ a $\$ 20,000$ & 43 & 25.7 \\
\hline$\$ 21,000$ a $\$ 30,000$ & 26 & 15.6 \\
\hline$\$ 31,000$ a $\$ 40,000$ & 11 & 6.6 \\
\hline$\$ 41,000$ a $\$ 50,000$ & 12 & 7.2 \\
\hline$\$ 51,000$ o más & 22 & 13.2 \\
\hline
\end{tabular}

\section{Instrumentos}

Para esta investigación se utilizaron tres medidas. Se utilizó un formulario para conocer las características sociodemográficas de la muestra. Se desarrolló un cuestionario para explorar de forma general las demandas tecnológicas y psicológicas que puedan estar experimentando los estudiantes activos estudiando desde sus casas. Este cuestionario contó con 42 preguntas cerradas que se contestaban en formato de Sí, No o No Aplica. Finalmente, utilizamos una escala que mide la ansiedad basada en el COVID-19 (González-Rivera et al, 2020; Lee, 2020). Esta escala cuenta con cinco reactivos que se contestan con un anclaje de respuesta tipo Likert de cinco puntos (Ninguna, Menos de un día o dos, Varios días, Más de 7 días, y Casi todos los días durante las últimas 2 semanas). La escala de ansiedad por COVID-19 demostró tener buena consistencia interna $(\alpha=.88)$.

\section{Estrategias en el Análisis de Datos}

Para realizar los análisis de esta investigación se utilizó el programa IBM SPSS Statistics (versión 26). Se realizaron análisis de distribuciones de frecuencia para cada una de las preguntas del cuestionario de Demandas Tecnológicas y Psicológicas en Estudiantes Virtuales. Esto con la finalidad de poder tener un panorama sobre cuáles demandas son las que los estudiantes perciben como que les están afectando mayormente. Además, se realizaron tablas cruzadas y se examinaron las diferencias significativas de las proporciones de las columnas en las tablas cruzadas mediante la prueba Chi-cuadrada con un nivel de significancia de $95 \%$, y se realizaron gráficas de barras sobre los análisis realizados en las tablas cruzadas.

\section{RESULTADOS}

La mayoría de los participantes de esta investigación indicaron que sus clases se movieron a modalidad en línea debido a la pandemia por COVID-19 $(98.2 \%, n=164)$. Poco más de la mitad de estos estudiantes $(51.5 \%, n=86)$ tuvieron experiencias previas tomando cursos en línea lo que presenta un cambio repentino de la experiencia educativa para el resto de los estudiantes que no tuvieron esa experiencia $(48.5 \%, n=81)$. El asunto se complica cuando observamos que la gran mayoría de los estudiantes de la muestra $(84.4 \%, n=141)$ no habían recibido adiestramientos, talleres, o explicaciones sobre lo que representa tomar cursos en línea durante el proceso de transición por la pandemia. En cuanto a la satisfacción por los cursos en línea la mayoría $(61.7 \%, n=103)$ indicaron que no se sentían satisfechos con esta modalidad educativa.

Entre las razones presentadas por los estudiantes ante la insatisfacción que sintieron por sus cursos en línea están:

1. falta de conocimiento de los profesores sobre cursos en línea,

2. sobrecarga de tareas,

3. problemas con la conexión de internet,

4. ausencia de respuesta a los mensajes y correos electrónicos por parte de los profesores, 
5. preferencia por cursos presenciales,

6. estresores del hogar,

7. estresores asociados a la pandemia,

8. dificultades para mantener la atención en los cursos en línea desde sus casas,

9. falta de organización y estructura correcta de los cursos en línea,

10. modificación de las prácticas instruccionales,

11. falta de recursos económicos y tecnológicos,

12. pobres destrezas tecnológicas,

13. falta de interacción social en los cursos en línea,

14. mayor complejidad en cursos en línea comparado a los presenciales,

15. mayor tiempo dedicado a tareas en línea comparado con las tareas asignadas en cursos presenciales,

16. e inflexibilidad de los profesores.

\section{Demandas Tecnológicas}

Al explorar las demandas tecnológicas que han experimentado los estudiantes de la muestra encontramos que la mayoría $(88.6 \%, n=148)$ sí tiene el equipo necesario para estudiar en línea. Asimismo, la mayoría de estos estudiantes $(82.8 \%, n=138)$ también indicaron que poseen el dominio tecnológico necesario para estudiar en línea. En cuanto al requerimiento de cámaras, poco más de la mitad de los estudiantes $(56.9 \%, n=95)$ indicaron que sus cursos les requieren que estén en vivo conectados por cámaras.

De igual manera, la gran mayoría de los estudiantes $(84.4 \%, n=141)$ expresaron que han tenido que aprender a utilizar nuevas tecnologías (aplicaciones, equipos, etc.) para poder realizar sus tareas académicas en línea. Sin embargo, la mayoría $(67.1 \%, n=112)$ no recibió apoyo tecnológico para poder realizar sus tareas en línea, tampoco recibieron apoyo tecnológico de parte de su universidad $(62.9 \%, n=105)$, ni de sus parejas o familiares $(49.1 \%, n=82)$. No obstante, sí recibieron apoyo tecnológico de parte de sus amistades $(62.3 \%, n=104)$. Además, la mayoría de los estudiantes $(79.6 \%, n=133)$ indicaron haber tenido problemas con su servicio de internet para realizar sus tareas académicas en línea.

\section{Demandas Académicas}

Como parte de las demandas académicas experimentadas por la muestra de estudiantes universi- tarios encontramos que la gran mayoría de estos $(83.2 \%, n=139)$ indicaron que su carga académica aumentó desde que se movieron a la modalidad en línea. Además, la mayoría $(74.3 \%, n=124)$ expresó que la exigencia académica no ha sido la misma que antes de la pandemia por COVID-19. Respecto al proceso de transición de la modalidad presencial a en línea, los estudiantes $(70.1 \%, n=117)$ expresaron que su universidad no les orientó sobre lo que conlleva estudiar en línea. Sin embargo, más de la mitad de los estudiantes $(65.3 \%, n=109)$ indicaron que sus universidades hicieron las modificaciones pertinentes para que sus clases se ajustaran a la modalidad en línea.

Respecto a los horarios de estudios, los estudiantes $(73.1 \%, n=122)$ indicaron que sus horas de estudio habían aumentado desde que estudian en línea. Además, la mayoría $(81.4 \%, n=136)$ también indicó que no se ha mantenido su horario de estudio igual desde que estudian en línea. Asimismo, los estudiantes $(87.4 \%, n=146)$ indicaron que sus agendas diarias se vieron alteradas desde que se movieron a estudiar en línea. No obstante, poco más de la mitad de los estudiantes $(57.5 \%, n=96)$ indicaron que tienen un horario establecido para estudiar en línea.

Por otro lado, se encontró que muchos estudiantes $(64.7 \%, n=108)$ indicaron que sienten que no pueden realizar sus tareas académicas sin problemas, y que se les hace complicado $(72.5 \%, n=121)$ estudiar en esta modalidad. No obstante, la mayoría de los estudiantes $(67.7 \%, n=113)$ indicaron que sienten el apoyo de sus familiares al estudiar en línea.

Poco más de la mitad de los participantes (51.5\%, $n=86$ ) indicaron que no han pensado en darse de baja de sus clases, sin embargo, el resto de los estudiantes $(48.5 \%, n=81)$ expresaron que sí pensaron darse de baja de sus clases. Sin embargo, la mayoría $(70.1 \%, n$ = 117) reportó que no han pensado cambiar de universidad. En cuanto al nivel de seguridad, poco más de la mitad (58.7\%, $n=98)$ indicaron que no se sentían seguros estudiando en línea. Respecto a las clases en línea, los estudiantes $(67.1 \%, n=112)$ expresaron que los mismos son menos flexibles que cuando las tomaban de forma presencial. Desde el aspecto de la salud mental, la mayoría de los estudiantes indicaron que sienten que su universidad no se preocupa por su seguridad emocional $(68.3 \%, n=114)$, ni de su salud mental $(66.5 \%, n=111)$. 


\section{Demandas Psicológicas}

Dentro de las demandas psicológicas experimentadas por la muestra de estudiantes universitarios pudimos observar que expresaron un aumento en sus niveles de estrés $(90.4 \%, n=151)$ y ansiedad $(83.8 \%, n$ $=140$ ). De igual forma, han expresado que el balance entre su vida familiar, laboral, y académica se han visto afectadas negativamente $(70.1 \%, n=117)$. Junto a esto, los estudiantes expresaron problemas para dormir recientemente $(80.2 \%, n=134)$, un problema que poco más de la mitad $(63.5 \%, n=106)$ indicó que no tenía antes de la pandemia por COVID-19. Además, encontramos que la mayoría de los estudiantes $(72.5 \%, n=121)$ reportó que no han podido desconectar sus pensamientos de sus clases una vez terminan de estudiar. Asimismo, los estudiantes (83.8\%, $n=$ 140) indicaron que se han sentido sin ánimos para estudiar durante la pandemia por COVID-19. La mayoría de los estudiantes $(79.0 \%, n=132)$ reportó que no se sienten productivos en sus clases durante la pandemia. No obstante, poco más de la mitad de los estudiantes $(51.5 \%, n=86)$ indicó que su calidad de vida es buena. Además, de aquellos estudiantes que tienen hijos, la mayoría $(29.9 \%, n=50)$ indicó que no tienen que ayudar a sus hijos con sus tareas en la casa.

Por otro lado, la mayoría de los participantes indicaron que sus creencias y prácticas religiosas se han mantenido luego de la pandemia por COVID-19 $(49.7 \%, n=83)$ al igual que sus creencias y prácticas espirituales $(53.9 \%, n=90)$. No obstante, cabe destacar que un grupo indicó que sus creencias y prácticas religiosas sí se modificaron luego de la pandemia $(17.4 \%, n=29)$ y de forma similar con sus creencias y prácticas espirituales $(17.4 \%, n=29)$. Parte de las dificultades que han expresado los estudiantes en esta investigación han sido problemas económicos (49.1\%, $n$ $=82$ ) y cambios en los hábitos alimenticios desde que estudian en línea $(79.0 \%, n=132)$, de los que reportaron ser menos saludables que antes $(70.7 \%, n=118)$.

\section{Satisfacción con Clases en Línea}

Para tener un entendimiento mejor sobre por qué algunos estudiantes indicaron satisfacción y otros expresaron insatisfacción por sus clases en línea, realizamos una tabla cruzada entre la satisfacción por las clases en línea y si tienen un horario establecido para estudiar en línea. El resultado obtenido demostró que el $71.9 \%$ de los estudiantes que están satisfechos con sus clases en línea también tienen un horario establecido para estudiar. Esta proporción fue estadísticamente significativa $(2(1)=8.792, p<.01)$ por lo que el tener un horario establecido puede contribuir positivamente a la satisfacción por las clases en línea junto con algunas variables adicionales como la comunicación, la organización del curso, y la experiencia del profesor con cursos en línea, entre otros.

Figura 1

Satisfacción con Clases en Línea y Horario Establecido para Estudiar en Línea.

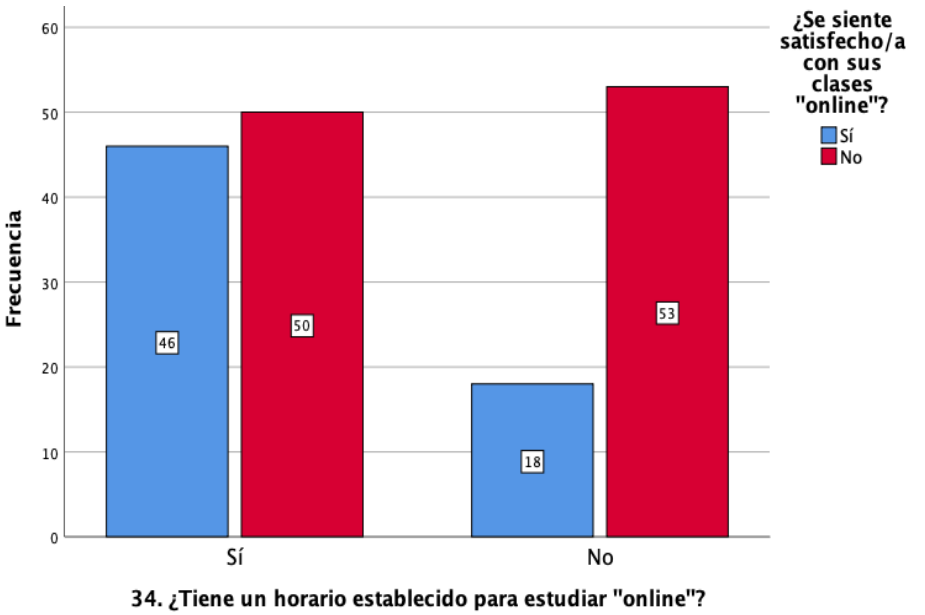

Finalmente, se realizó una prueba U de MannWhitney para examinar si hay diferencias significativas en el nivel de ansiedad por COVID-19 por la satisfacción de las clases en línea. El resultado obtenido indica que no hay diferencias significativas entre la satisfacción por las clases en línea (sí o no) y el nivel de ansiedad por COVID-19 en la muestra de estudiantes, $U=3,830, S E=299.21, Z=1.785, p=.074$. Se concluye que satisfacción por las clases en línea no se ve afectada de forma significativa por la ansiedad por COVID-19 en la muestra de estudiantes. Sin embargo, cabe destacar que las puntuaciones de la medida de ansiedad por COVID-19 fluctuó entre 1 a 5 con una media de $1.88(D E=0.96)$.

\section{DISCUSIÓN}

El propósito de esta investigación fue examinar las demandas tecnológicas, académicas y psicológicas experimentadas por los estudiantes universitarios durante la pandemia por COVID-19 en Puerto Rico. Antes de discutir nuestros hallazgos nos parece pertinente contextualizar cómo fue la transición de la modalidad presencial a e-learning durante el mes de 
marzo 2020. Todas las instituciones universitarias del país brindan la mayoría de su oferta académica de forma presencial. Muchas utilizan sistemas de gestión de aprendizaje como Blackboard, Canvas o Moodle como complemento de los cursos presenciales, pero evidentemente no son las herramientas principales de los mismos. Esto, a pesar que en los últimos años se han propuesto distintos modelos de enseñanza a distancia como aula invertida (curso híbrido) y aprendizaje electrónico (asincrónico o sincrónico). Nuestros resultados plasman esta realidad dado que prácticamente la mitad de los participantes no habían tomado cursos a distancia antes del COVID-19.

Con este perfil y ante una nueva realidad pandémica, el 100\% de las instituciones académicas, muy responsablemente desde la perspectiva salubrista, anunciaron a mediados de marzo 2020 el cierre de sus instalaciones físicas y la movilización de los cursos a modalidad a distancia. Este cambio abrupto y repentino -prácticamente fue de un día para otro- en un semestre bastante adelantado, forzó la implementación inmediata de modelos de aprendizaje a distancia. Al parecer, dicha transición no fue exitosa ni efectiva dado que más de la mitad de los estudiantes indicaron que no se sentían satisfechos con la modalidad educativa a distancia implementada por las universidades. Entre las razones principales de insatisfacción se encuentran: la falta de conocimiento de los profesores sobre la modalidad a distancia, la sobrecarga de tareas más allá de lo que estaba originalmente estipulado en el curso y problemas con la conexión de internet.

Nos pareció interesante que la insatisfacción no se debió a la falta de equipo electrónico -aunque algunos presentan esta necesidad- ni a la falta de competencia para manejar los mismos. Tampoco se vio afectada por los niveles de ansiedad por coronavirus, dado que el promedio de esta medida en los participantes fue 1.88 ( $\mathrm{DE}=0.96)$, y la puntuación diagnóstica de este instrumento es 10 (González-Rivera et al., 2020). Podemos decir entonces que, en general, los estudiantes de la muestra no se vieron afectados por ansiedad relacionada al COVID-19, sino que su ansiedad fue específica por la carga académica de sus cursos en línea y las demandas tecnológicas y académicas que experimentaron. No obstante, nos pareció preocupante que la mayoría de los estudiantes indicara que sienten que su universidad no se preocupa por su seguridad emocional. En términos de posibles factores protectores, nuestros hallazgos demostraron que tener un horario establecido de estudios puede ayudar a sentir mayor satisfacción en los cursos en línea y minimizar la carga percibida de trabajo.

Los resultados de nuestro estudio tienen varias implicaciones prácticas que podemos traducir en recomendaciones concretas para las instituciones académicas. Esperamos que las mismas sean bien recibidas por la academia y ayuden a promover un mayor bienestar organizacional en las comunidades universitarias dado que el semestre de agosto-diciembre 2020 también será en modalidad a distancia en la mayoría de las instituciones. Esto, con el fin de proporcionar un ambiente educativo ajustado a las necesidades tecnológicas, académicas y psicológicas que enfrenta toda la comunidad universitaria.

\section{Recomendaciones para las Universidades}

En primer lugar, dado el alto porciento de participantes $(84.4 \%)$ que no recibieron talleres o adiestramientos sobre lo que representa tomar cursos en línea, recomendamos que las instituciones educativas del país desarrollen un programa de capacitación para adiestrar estudiantes y facultad en los diversos procesos técnicos y tecnológicos que involucran la educación en línea. Esta serie de talleres deben ser ofrecidos antes de comenzar el próximo semestre académico. Además, debe existir un espacio de tiempo donde los estudiantes y profesores tengan la oportunidad de realizar preguntas referentes a sus cursos y al plan académico de la institución.

Segundo, en términos administrativos, recomendamos a los directores de los programas académicos que junto a su equipo de trabajo organicen la secuencia de cursos antes de que comiencen los mismos. Es decir, que los directores de los programas académicos, ordenen a la facultad de dicho programa que realicen una plantilla instruccional (puede ser en formato de tabla) donde expresen lo que se ofrecerá durante el semestre junto con toda la información pertinente como, por ejemplo, el calendario académico del curso, la descripción de las unidades, los objetivos, actividades, modalidad, evaluación, fecha de entrega, etc. Esta recomendación, debe realizarse antes del comienzo del semestre académico para que los estudiantes puedan tener una idea sobre el contenido del curso y logren organizarse acorde al mismo. Esta 
recomendación está fundamentada en la insatisfacción con los cursos en línea reportada por la mayoría de los participantes del estudio.

Tercero, a nivel de servicio, recomendamos a los directores de cada programa académico que estipulen un horario que fluctúe entre 24 a 36 horas para que los facultativos respondan los correos electrónicos de sus estudiantes. La comunicación entre estudiante y facultad es de suma importancia ya que en el transcurso del semestre académico pueden surgir dudas técnicas o académicas referentes al curso. Estas dudas deben ser contestadas lo antes posible para que los estudiantes puedan cumplir con el tiempo estipulado de las tareas y sientan la accesibilidad del profesor. Al momento, la comunicación es una herramienta esencial para el curso.

Cuarto, recomendamos a los profesores que consideren ser mas flexibles con los tiempos de entrega de asignaciones y trabajos, sin que esto afecte la calidad y rigurosidad de la enseñanza. Según los datos obtenidos, observamos que existen dificultades técnicas que, en la mayoría de los casos, están fuera del control de los estudiantes como, por ejemplo, pérdida de internet, problemas con la energía eléctrica, fallo de los dispositivos y pérdida de señal celular. La flexibilidad y la constante comunicación, tanto del profesor como del estudiante, son fundamentales en la solución de los pormenores que se presenten durante e semestre. Además, si un estudiante presenta dificultades técnicas o tecnológicas al momento de entregar una tarea, el profesor puede optar por recomendarle al estudiante que realice la tarea a mano y envíe la misma por foto al correo electrónico institucional utilizando su dispositivo móvil. El plan de flexibilidad de cada profesor no debe promover la irresponsabilidad en los estudiantes, por lo cual, estos arreglos deben estar suscritos a claras pautas de exigencia a los estudiantes, entre las cuales la primera deber ser una comunicación abierta por parte del estudiantado.

Quinto, en términos de bienestar, recomendamos que los profesores al inicio de cada curso permitan un espacio controlado de cinco a diez minutos para que los estudiantes expresen sus pensamientos dudas o comentarios para con el curso. El profesor puede desarrollar ciertas normas antes de proveer el espacio. No se trata de consejería, si no que el estudiante sienta que se le escucha y se le brinda espacio para expresarse sobre asuntos relacionados al curso. La mayoría de los cursos que se estarán ofreciendo el próximo semestre fueron desarrollados originalmente de manera presencial. Es por esto, que ese tiempo de expresión, que ocurre con más facilidad en un ambiente cara a cara, se pueda dar también en un ambiente electrónico, ya sea sincrónico o asincrónico.

Por último, si la facultad utiliza un sistema de gestión del aprendizaje (e.g., Blackboard, Canvas, Moodle, etc.), recomendamos que les permitan a sus estudiantes acceso abierto a las sesiones que fueron ofrecidas de forma sincrónica. De esta manera, si al estudiante le surge algún problema técnico o alguna emergencia, el mismo puede acceder a la sesión de la clase posteriormente y no pierde el contenido de la misma. Esta herramienta no es para dar licencia a los estudiantes para que se ausenten a las sesiones que son sincrónicas. Es por ello, que el profesor al inicio de la sesión académica debe establecer las normas a seguir en caso de ausencia. Esta recomendación tiene que ser cónsona con las políticas de asistencia de cada institución académica.

\section{Limitaciones y Futuras Investigaciones}

Las principales limitaciones del estudio están relacionadas con el tipo de muestreo utilizado (por disponibilidad) y el tamaño de la muestra $(n=167)$. Este tipo de muestreo hace que la generalización de los resultados sea imposible, lo que significa que nuestros hallazgos son relevantes solo a los estudiantes universitarios que participaron de este estudio. Futuras investigaciones de esta índole deben aumentar la muestra significativamente. La dificultad para reclutar participantes puede estar relacionada con la misma situación del estudiantado en la pandemia y la sobrecarga de tareas académicas que reportaron tener en este estudio. La quemazón por tanto trabajo puede hacer que el estudiantado este apático a participar de estudios e investigaciones en línea. Por último, la naturaleza descriptiva de nuestro estudio no permite establecer relaciones causales entre las variables estudiadas.

\section{CONCLUSIÓN}

En esta investigación pudimos observar las demandas tecnológicas, académicas, y psicológicas que le aquejaron a la muestra de estudiantes universitarios recopilada. Nuestros hallazgos son un pequeño 
reflejo de las consecuencias que traen los cambios abruptos de modalidades educativas presenciales a virtuales durante un semestre académico activo en los estudiantes universitarios, en este caso, a consecuencia de la pandemia por COVID-19. Es importante que se continúe indagando sobre las demandas tecnológicas, académicas y psicológicas que puedan experimentar los estudiantes bajo escenarios de cambios repentinos para ampliar el conocimiento al respecto. Esperamos que esta investigación abra paso para explorar estas situaciones y sus efectos más

\section{REFERENCIAS}

Area, M., \& Adell, J. (2009). e-Learning: Enseñar y aprender en espacios virtuales. En J. De Pablos (Ed.), Tecnología Educativa. La formación del profesorado en la era de Internet (pp. 391-424). Aljibe.

Balluerka-Lasa, N., Gómez-Benito, J., Hidalgo-Montesinos, D., Gorostiaga-Manterola, A., Espada-Sánchez, J. P., Padilla-García, J. L., \& Santed-Germán, M. A. (2020). Las consecuencias psicológicas de la COVID-19 y el confinamiento: Informe de investigación. Universidad del País Vasco: España. https://www.ub.edu/web/ub/ca/menu_eines/noticies/docs/Consecuencias_psicologicas_COVID-19.pdf

Banco Interamericano de Desarrollo. (2020). La educación en tiempos del coronavirus: Los sistemas educativos de América Latina y el Caribe ante COVID-19. División de Educación. https://publications.iadb.org/publications/spanish/document/La-educacion-entiempos-del-coronavirus-Los-sistemas-educativos-de-America-Latina-y-el-Caribe-ante-COVID-19.pdf

BBC News Mundo. (2020). Coronavirus: El mapa que muestra el número de infectados y muertos en el mundo por COVID-19. https://www.bbc.com/mundo/noticias-51705060

González-Rivera, J. A., Rosario-Rodríguez, A., \& Cruz-Santos, A. (2020). Escala de Ansiedad por Coronavirus: Un nuevo instrumento para medir síntomas de ansiedad asociados al COVID-19. Artículos sometido para publicación.

Hernández-Sampieri, R., \& Mendoza, C. P. (2018). Metodología de la investigación: Las rutas cuantitativa, cualitativa y mixta. McGrawHill.

Instituto Internacional para la Educación Superior en América Latina y el Caribe. (2020). COVID-19 y educación superior: De los efectos inmediatos al día después. Organización de las Naciones Unidas para la Educación, la Ciencia y la Cultura. http://www.iesalc.unesco.org/wp-content/u ploads/2020/05/COVID-19-ES-130520.pdf

Landeta, A. (2007). Buenas Prácticas de e-learning. ANCED.

Lee, S. (2020). Coronavirus Anxiety Scale: A brief mental health screener for COVID-19 related anxiety. Death Studies, 44(7), 19. https://doi.org/10.1080/07481187.2020.1748481

Li, Q., Guan, X., Wu, P., Wang, X., Zhou, L., Tong, Y., Ren, R., Leung, K., Lau, E., Wong, J. Y., Xing, X., Xiang, N., Wu, Y., Li, C., Chen, Q., Li, D., Liu, T., Zhao, J., Liu, M., Tu, W., ... Feng, adelante en la educación superior, además, de que sirva para que las universidades incluyan en sus planes de emergencia y contingencia protocolos que consideren el impacto de los cambios inmediatos de modalidades educativas en sus estudiantes y puedan prevenirlas.

Financiamiento: La presente investigación no fue financiada por alguna entidad ni patrocinador.

Conflicto de Intereses: No existen conflictos de intereses de parte de los autores de la investigación.

Z. (2020). Early Transmission Dynamics in Wuhan, China, of Novel Coronavirus-Infected Pneumonia. The New England journal of medicine, 382(13), 1199-1207. https://doi.org/10.1056/NEJMoa2001316

Mezzadra, F., \& Bilbao, R. (2010). Las nuevas tecnologías de la información y la comunicación en educación: discusiones y opciones de política educativa. Fundación CIPPEC.

Monasterio, D., \& Briceño, M. (2020). Educación mediada por las Tecnologías: Un desafío ante la coyuntura del Covid-19. Observador del Conocimiento, 5(1), 136-148. http://www.oncti.gob.ve/ojs/index.php/rev_ODC/article/view/31

Murphy, E., Rodríguez-Manzanares, M. A., \& Barbour, M. (2011). Asynchronous and synchronous online teaching: Perspectives of Canadian high school distance education teachers. British Journal of Educational Technology, 42(4), 583-591. https://doi.org/10.1111/j.1467-8535.2010.01112.x

Páez-Barón, E. M., Corredor-Camargo, E. S., \& Fonseca-Carreño, J. A. (2015). Evaluación del uso de herramientas sincrónicas y asincrónicas en procesos de formación de las ciencias agropecuarias. Revista Ciencia y Agricultura, 13(1), 77-90. https://dialnet.unirioja.es/descarga/articulo/5560523.pdf

Regmi, K., Jones, L. (2020). A systematic review of the factors -enablers and barriers- affecting e-learning in health sciences education. BMC Medical Education, 20(91), 1-18 https://doi.org/10.1186/s12909-020-02007-6

RISE. (2020). A Survey for Students affected by coronavirus. https://risefree.org/covid-19-help/

Saini, A. (2020). Exploring the potential of digital technology in achieving quality education: Rethinking pedagogy. Poster presented in 14th International Technology, Education and Development Conference; Valencia, Spain.

Sanz, I., Sáinz, J., \& Capilla, A. (2020). Efectos de la crisis del coronavirus en la educación. Organización de Estados Iberoamericanos para la Educación, la Ciencia y la Cultura (OEI).

UNESCO. (2019). Rethinking pedagogy: Exploring the potential of digital technology in achieving quality education. Mahatma Gandhi Institute of Education for Peace and Sustainable Development. https://d27gr4uvgxfbqz.cloudfront.net/files\%2Fe7f932d8- 
2379-4e9a-8922-45a2297a6137_Rethinking\%20Pedagogy.pdf

UNESCO. (2020, April). 290 million students out of school due to COVID-19: UNESCO releases first global numbers and mobilizes response. https://en.unesco.org/news/290-million-students-out-school-due-covid-19-unesco-releases-first-globalnumbers-and-mobilizes

Uprichard, K. (2020). E-learning in a new era: enablers and barriers to its implementation in nursing. British Journal of Community Nursing, 25(6), 272-275. http://doi.org/10.12968/bjen.2020.25.6.272

Wang, W., Xu,Y., Gao, R., Lu, R., Han, K., Wu, G., \& Tan, W. Detection of SARS-CoV-2 in different types of clinical specimens. JAMA， 323(18), 1843-1844. http://jamanetwork.com/article.aspx?doi=10.1001/jama.2020.3786
Wang Y., Zhang D., Du G., Du R.,Zhao J., Jin Y., Fu S., Gao L., Cheng Z.,Lu Q., Hu Y., Luo G., Wang K., Lu Y., Li H., Wang S., Ruan S., Yang C., Mei C., Wang Y., Ding D., Wu F., Tang X., Ye X., Ye Y., Liu B., Yang J., Yin W., Wang A., Fan G., Zhou F., Liu Z., Gu X., Xu J., Shang L., Zhang Y., Cao L., Guo T., Wan Y, Qin H., Jiang Y., Jaki T., Hayden F., Horby P., Bin Cao B., \& Chen Wang. (2020). Remdesivir in adults with severe COVID-19: A randomised, double-blind, placebo-controlled, multicentre trial. The Lancet, 395 (10236), 1569-1578. https://doi.org/10.1016/S0140-6736(20)31022-9

Yin, Y., \& Wunderink, R. G. (2018). MERS, SARS and other coronaviruses as causes of pneumonia. Respirology (Carlton, Vic.), 23(2), 130-137. https://doi.org/10.1111/resp.13196

Obra bajo licencia de Creative Commons Atribución 4.0 Internacional (CC BY 4.0). (C) 2020 Autores. 